\title{
COPRIME ACTIONS WITH ALL ORBIT SIZES SMALL
}

\author{
DAVID GLUCK
}

(Communicated by Pham Huu Tiep)

\begin{abstract}
Let a $p^{\prime}$-group $G$ act faithfully on a finite $p$-group $P$. Suppose that every $G$-orbit on $P$ has size at most $p-1$. We show that $G$ must have a regular orbit on $P$.
\end{abstract}

\section{INTRODUCTION}

Let $G$ be a $p^{\prime}$-group which acts faithfully on a finite $p$-group $P$. Suppose that all orbit sizes of $G$ on $P$ are "small" in terms of $p$. Must it follow, somewhat paradoxically, that $G$ has a regular orbit on $P$ ?

I. M. Isaacs [3, Theorem 3.1] showed that if all orbit sizes are at most $(p+1) / 2$, then $G$ must indeed have a regular orbit on $P$, and he gave an example showing that if there is an orbit of size $p+1$, then there need not be a regular orbit. He asks if there must be a regular orbit if the bound $(p+1) / 2$ is replaced by $p-1$. This would be best possible; $p$ cannot be an orbit size because $(|G|, p)=1$. We answer his question in the affirmative:

Theorem A. Let $G$ be a $p^{\prime}$-group that acts faithfully on a finite p-group P. Suppose that every $G$-orbit on $P$ has size at most $p-1$. Then $G$ has a regular orbit on $P$.

We remark that the coprimeness hypothesis in Theorem A is appropriate but logically superfluous; if $x \in G$ had order $p$, then $\langle x\rangle$ would have an orbit of size $p$, so some $G$-orbit would have size at least $p$.

We prove Theorem A by a variety of counting arguments; the simple group classification is not needed. We reduce to the case that $P$ is elementary abelian and irreducible under the action of $G$. We then show that if the proportion of pseudoreflections in $G$ is at most $1 / 3$, then $G$ must have a regular orbit on $P$. We quickly deduce that regular orbits must exist when $|P|>p^{3}$. More detailed analysis is needed when $|P| \leq p^{3}$.

\section{The General CASE}

In this section we reduce Theorem A to the case that $P$ is elementary abelian and $G$-irreducible. We introduce the important pseudoreflection ratio $\mathrm{r}(G, V)$ for coprime linear groups. We show that if $\mathrm{r}(G, V) \leq 1 / 3$, then $(G, V)$ is not a counterexample to Theorem A. In particular, $(G, V)$ is not a counterexample to Theorem A if $V$ is an irreducible $\operatorname{GF}(p)[G]$-module of dimension greater than 3 .

Received by the editors September 15, 2013 and, in revised form, January 7, 2014.

2010 Mathematics Subject Classification. Primary 20D45; Secondary 20H30.

Key words and phrases. Coprime actions, orbit sizes, pseudoreflections. 
Lemma 2.1. Suppose $G$ acts faithfully on a finite p-group $P$. Suppose that $P$ is a nontrivial direct product $P_{1} \times P_{2}$ of $G$-invariant subgroups. Suppose that $G$ acts nontrivially on $P_{1}$ and on $P_{2}$ and that every $G$-orbit on $P$ has size at most $p+1$. Then $G$ has a regular orbit on $P$.

Proof. This is a slightly weaker form of [3, Theorem 3.3].

Lemma 2.2. If Theorem $A$ is false, then there is a counterexample in which $P$ is elementary abelian and irreducible under the action of $G$.

Proof. Suppose $(G, P)$ is a counterexample to Theorem A. Let $A=P / \Phi(P)$. Then $G$ acts faithfully on $A$ and each $G$-orbit on $A$ has size at most $p-1$. Since $G$ has no regular orbit on $P$, it follows that $G$ has no regular orbit on $A$. Thus $(G, A)$ is a counterexample to Theorem A.

We have $A=[A, G] \times \mathrm{C}_{A}(G)$. Now $G$ acts faithfully on $[A, G]$, so $(G,[A, G])$ is a counterexample to Theorem $\mathrm{A}$. We may therefore assume that $A=[A, G]$. Then if $A$ is a nontrivial direct sum of $G$-invariant subgroups, then $G$ acts nontrivially on both summands. By Lemma 2.1, $G$ has a regular orbit on $A$, contrary to hypothesis. We conclude that $A$ has no nontrivial $G$-invariant direct summand. Since $(|G|,|A|)=1$, we conclude that $A$ is $G$-irrreducible.

Definition 2.3. Let $V$ be a $d$-dimensional vector space over $\operatorname{GF}(p)$. We say that a $p^{\prime}$-element $g \in \mathrm{GL}(V)$ is a pseudoreflection if $\left|\mathrm{C}_{V}(g)\right|=p^{d-1}$. Note that this implies that $g$ is diagonalizable over $\operatorname{GF}(p)$. If $G$ is a subgroup of $\operatorname{GL}(V)$, we define $\mathrm{r}(G, V)$ to be $|G|^{-1}$ times the number of pseudoreflections in $G$.

Proposition 2.4. Let $V$ be a finite vector space of characteristic $p$. Let $G \leq \mathrm{GL}(V)$ be a $p^{\prime}$-subgroup of order $n$. Suppose that every $G$-orbit on $V$ has size at most $p-1$ and suppose that $r(G, V) \leq 1 / 3$. Then $n<3 p / 2$ and $G$ has a regular orbit on $V$.

Proof. Let $S$ be the set of all ordered pairs $(v, g) \in V \times G$ such that $v g=v$. On the one hand,

$$
|S|=\sum_{v \in V}\left|\mathrm{C}_{G}(v)\right| \geq \sum_{v \in V} n /(p-1)=n|V| /(p-1) .
$$

On the other hand, since $|S|=\sum_{g \in G}\left|\mathrm{C}_{V}(g)\right|$, the hypotheses give

$$
|V|+(n / 3)|V| / p+((2 n / 3)-1)|V| / p^{2} \geq|S| \geq n|V| /(p-1) .
$$

Hence

$$
1+n / 3 p+((2 n / 3)-1) / p^{2} \geq n /(p-1) .
$$

For fixed $p$, both sides of $(*)$ are linear functions of $n$. Since $1 / 3 p+2 / 3 p^{2}<1 /(p-1)$ for all $p$, the desired conclusion that $(*)$ is false for all $n \geq 3 p / 2$ will follow if we show that $(*)$ is false for $n=3 p / 2$.

Suppose then that $n=3 p / 2$, so that $(*)$ becomes

$$
1+1 / 2+(p-1) / p^{2} \geq 3 / 2+3 / 2(p-1),
$$

which simplifies to $(p-1)^{2} \geq 3 p^{2} / 2$, which is false for all $p$, as desired. Hence $n<3 p / 2$. 
To show that $G$ has a regular orbit on $V$, it suffixes to show $|S|=\sum_{g \in G}\left|\mathrm{C}_{V}(g)\right|$ is less than $2|V|$. Since $\mathrm{r}(G, V) \leq 1 / 3$ and $n<3 p / 2$, we have

$$
|S|<|V|\left(1+n / 3 p+2 n / 3 p^{2}\right)<|V|(1+1 / 2+1 / p) \leq 2|V|,
$$

as desired.

Corollary 2.5. Let $V$ be a vector space of dimension $d>3$ over $G F(p)$. Let $G \leq \mathrm{GL}(V)$ be an irreducible $p^{\prime}$-group such that every $G$-orbit on $V$ has size at most $p-1$. Then $G$ has a regular orbit on $V$.

Proof. Suppose, to get a contradiction, that $\mathrm{r}(G, V)>1 / 4$. Then, in particular, $\mathrm{r}(G, V)>0$, and so $\mathrm{C}_{V}(g)$ is a hyperplane in $V$ for some $g \in G$. Since $V / \mathrm{C}_{V}(g)$ is a vector space over $\operatorname{End}_{\mathrm{GF}(p)[G]}(V)$, it follows that $\operatorname{End}_{\mathrm{GF}(p)[G]}(V)=\mathrm{GF}(p)$, and so $V$ is an absolutely irreducible $\mathrm{GF}(p)[G]$-module. It follows that the Brauer character $\chi$ of $V$ is an irreducible complex character of $G$.

If $g \in G$ centralizes a hyperplane in $V$, then $|\chi(g)| \geq d-2$. Since $\mathrm{r}(G, V)>1 / 4$, we have

$$
|G|=\sum_{g \in G}|\chi(g)|^{2}>(|G| / 4)(d-2)^{2} .
$$

Since $d \geq 4$, we have $(d-2)^{2} \geq 4$, and so $|G|>|G|$, a contradiction. We conclude that $\mathrm{r}(G, V) \leq 1 / 4$. By Proposition 2.4, $G$ has a regular orbit on $V$.

\section{LOW DIMENSIONS}

Throughout this section we assume that $V$ is a faithful irreducible $\operatorname{GF}(p)[G]$ module of cardinality $p^{d}$, with $1<d \leq 3$. We assume that $G$ is a $p^{\prime}$-group and that every $G$-orbit on $V$ has size at most $p-1$. Our goal is to show that $G$ has a regular orbit on $V$.

Lemma 3.1. Suppose $G$ has no regular orbit on $V$. Then $V$ is an absolutely irreducible $G F(p)[G]$-module.

Proof. Let $E=\operatorname{End}_{\mathrm{GF}(p)[G]}(V)$. Then $V$ is an absolutely irreducible $E[G]$-module. Since $d$ is prime, we have $E=\mathrm{GF}(p)$ or $E=\mathrm{GF}\left(p^{d}\right)$. In the former case, $V$ is an absolutely irreducible $\operatorname{GF}(p)[G]$-module. In the latter case, $V$ has dimension 1 over $E$ and every nonzero vector in $V$ lies in a regular $G$-orbit.

Lemma 3.2. Suppose $G$ has no regular orbit on $V$. Then $|\mathrm{Z}(G)|<3$ if $d=3$ and $|\mathrm{Z}(G)|<6$ if $d=2$.

Proof. Let $k=|G: \mathrm{Z}(G)|$ and let $\left\{g_{1}, \ldots, g_{k}\right\}$ be a set of coset representatives for $\mathrm{Z}(G)$ in $G$. Fix $i \leq k$. Lemma 3.1 implies that every element of $\mathrm{Z}(G)$ acts on $V$ as multiplication by some scalar in $\operatorname{GF}(p)^{*}$. If $d=3$, it follows that at most one element of the coset $g_{i} \mathrm{Z}(G)$ can have 1 as a double eigenvalue on $V$. Thus $\mathrm{r}(G, V) \leq k /|G|=1 /|\mathrm{Z}(G)|$. If $d=2$, then at most two elements of the coset $g_{i} \mathrm{Z}(G)$ can have 1 as an eigenvalue on $|V|$. Hence $G$ contains at most $2 k$ pseudoreflections and so $\mathrm{r}(G, V) \leq 2 k /|G|=2 /|\mathrm{Z}(G)|$. The conclusion of the lemma now follows from Proposition 2.4 .

Lemma 3.3. Suppose a normal subgroup $A$ of $G$ of index $d$ acts Frobeniusly on $V$. Then $G$ has a regular orbit on $V$. 
Proof. Since $A$ has a regular orbit on $V$, the hypotheses for this section imply that $|A| \leq p-1$. Suppose first that $d=2$. Then $|G-A| \leq p-1$, so the number of nonzero vectors in $V$ fixed by some nonidentity element of $G$ is at most $(p-1)(p-1)<p^{2}-1$. Hence $G$ has a regular orbit on $V$. Suppose next that $d=3$. Fix a coset $C$ of $A$ with $C \neq A$. Then every nonzero vector in $V$ that is fixed by a nonidentity element of $G$ is fixed by an element of $C$. Since $|C| \leq p-1$, the number of nonzero vectors in $V$ fixed by some nonidentity element of $G$ is at most $(p-1)\left(p^{2}-1\right)<p^{3}-1$. Hence $G$ has a regular orbit on $V$, as desired.

Lemma 3.4. Suppose that $G$ is not solvable. Then $G=Q \mathrm{Z}(G)$ for a quasisimple group $Q$. If $d=2$, then $Q$ is $\mathrm{SL}_{2}(5)$. If $d=3$, then $Q$ is $\mathrm{A}_{5}, \mathrm{~L}_{2}(7)$, or $3 . \mathrm{A}_{6}$.

Proof. By Lemma 3.1, $G$ acts coprimely and absolutely irreducibly on $V$. Hence $G$ is isomorphic to a complex linear group of degree $d \leq 3$. An easy abstract argument (see [2, Lemma 1.1]) shows that $G / \mathrm{Z}(G)$ is contained in the automorphism group of a simple group. By [5, pp. 213, 250-251], $G / \mathrm{Z}(G)$ is in fact simple of order 60,168 , or 360. Hence $G=\mathrm{F}^{*}(G)=Q \mathrm{Z}(G)$ for a quasisimple group $Q$. The possibilities for $Q$ can then be determined from [1].

Lemma 3.5. Suppose $G$ is not solvable. Then $G$ has a regular orbit on $V$.

Proof. First suppose $d=3$. Let $G=Q \mathrm{Z}(G)$ as in Lemma 3.4. By Lemma 3.2, we may assume $|\mathrm{Z}(G)|<3$. Hence $G$ is $\mathrm{A}_{5}, \mathrm{~L}_{2}(7), \mathrm{A}_{5} \times \mathrm{Z}_{2}$, or $\mathrm{L}_{2}(7) \times \mathrm{Z}_{2}$. Since $\mathrm{A}_{5}$ and $\mathrm{L}_{2}(7)$ are contained in $\mathrm{SL}(V)$, we have $\mathrm{r}\left(\mathrm{A}_{5}, V\right)=\mathrm{r}\left(\mathrm{L}_{2}(7), V\right)=0$. If $g \in \mathrm{A}_{5}$ and $\langle z\rangle=\mathrm{Z}\left(\mathrm{A}_{5} \times \mathrm{Z}_{2}\right)$, then $g z$ is a pseudoreflection only if $g$ is an involution. Hence $\mathrm{A}_{5} \times \mathrm{Z}_{2}$ contains at most 15 pseudoreflections and so $\mathrm{r}\left(\mathrm{A}_{5} \times \mathrm{Z}_{2}\right) \leq 15 / 120<1 / 3$. Similarly, $\mathrm{r}\left(\mathrm{L}_{2}(7) \times \mathrm{Z}_{2}\right) \leq 21 / 336<1 / 3$. In all cases $G$ has a regular orbit on $V$ by Proposition 2.4.

Suppose next that $d=2$. By Lemma 3.4 and Lemma 3.2, we have $G=\mathrm{SL}_{2}(5)$ or $G=\mathrm{SL}_{2}(5) * \mathrm{Z}_{4}$. Since $\mathrm{SL}_{2}(5) \leq \mathrm{SL}(V)$, we have $\mathrm{r}\left(\mathrm{SL}_{2}(5), V\right)=0$. Suppose then that $G=\mathrm{SL}_{2}(5) * \mathrm{Z}_{4}$. Let $\mathrm{Z}(G)=\langle z\rangle$ and let $g \in \mathrm{SL}_{2}(5)$. Then $g z$ is a pseudoreflection if and only if $g$ has order 4 . Since $\mathrm{SL}_{2}(5)$ contains exactly 30 elements of order 4 , we have $\mathrm{r}(G, V) \leq 30 / 240<1 / 3$. In either case $G$ has a regular orbit on $V$ by Proposition 2.4.

Proposition 3.6. Suppose that $G$ acts primitively on $V$. Then $G$ has a regular orbit on $V$.

Proof. Suppose that $G$ is a counterexample to Proposition 3.6. By Lemma 3.5, $G$ is solvable. Let $A$ be a normal abelian subgroup of $G$. We claim that $A \leq \mathrm{Z}(G)$. If $V_{A}$ is reducible, then, since $d$ is prime, $V_{A}$ is a direct sum of one-dimensional submodules. Since $V_{A}$ is homogeneous and $G$ acts faithfully on $V$, we have $A \leq$ $\mathrm{Z}(G)$, as desired. Hence we may assume that $V_{A}$ is irreducible. By [4, Theorem 2.1], $G$ is contained in the semilinear group $\Gamma\left(p^{d}\right)$. Thus $G$ has a normal cyclic subgroup of index $d$ that acts Frobeniusly on $V$. By Lemma 3.3, $G$ has a regular orbit on $V$, contrary to hypothesis. This proves the claim.

By [4, Corollary 1.10(i,x)], we therefore have $\mathrm{F}(G)=E \mathrm{Z}(G)$, where $E \triangleleft G$. By the preceding paragraph, we may assume that $E$ has no cyclic Sylow subgroups. Hence $E$ is extraspecial by [4, Corollary 1.10(ii)]. Since $E$ has a faithful irreducible representation of degree $d$, we have $|E|=d^{3}$. Suppose $d=3$. Then $\mathrm{Z}(E) \leq \operatorname{GF}(p)^{*}$, since $E$ is absolutely irreducible on $V$. Hence $\mathrm{Z}(E) \leq \mathrm{Z}(G)$ and so $|\mathrm{Z}(G)| \geq 3$. By Lemma $3.2, G$ has a regular orbit on $V$, contrary to hypothesis. 
Hence $d=2$. If $G$ were nilpotent, then $G=\mathrm{F}(G)=E Z(G)$ would have a normal abelian subgroup $A$ of index 2. As above, $A \leq \mathrm{Z}(G)$ and so $|G: \mathrm{Z}(G)|=2$, which is impossible. Hence $G$ is not nilpotent. It follows that $E \cong \mathrm{Q}_{8}$ and $G / \mathrm{Z}(G)$ is isomorphic to $A_{4}$ or $S_{4}$.

By Lemma 3.2, we have $|\mathrm{Z}(G)|<6$. Hence $|\mathrm{Z}(G)|=2$ or $|\mathrm{Z}(G)|=4$. Since $(3,|\mathrm{Z}(G)|)=1, G$ contains an element of order 3 which acts nontrivially on $E$. Hence $G$ contains an $\mathrm{SL}_{2}(3)$ subgroup and $G$ contains a subgroup $S$ of index 1 or 2 with $S \cong \mathrm{SL}_{2}(3)$ if $|\mathrm{Z}(G)|=2$ and $S \cong \mathrm{SL}_{2}(3) * \mathrm{Z}_{4}$ if $|\mathrm{Z}(G)|=4$.

Suppose that $G=S$. We claim that $\mathrm{r}(G, V) \leq 1 / 3$. Indeed if $|\mathrm{Z}(G)|=2$, so that $S \cong \mathrm{SL}_{2}(3)$, then only elements of order 3 in $G$ can be pseudoreflections. Since $G$ has exactly 8 such elements, the claim follows. If $|\mathrm{Z}(G)|=4$, so that $G \cong \mathrm{SL}_{2}(3) * \mathrm{Z}_{4}$, then $\mathrm{SL}_{2}(3)$ contains at most 8 pseudoreflections as above. If $g \in \mathrm{SL}_{2}(3)$ and $z \in \mathrm{Z}(G)$ has order 4 , then $g z$ can be a pseudoreflection only if $g$ has order 4 . Hence the coset $\mathrm{SL}_{2}(3) z$ contains at most 6 pseudoreflections. Thus $\mathrm{r}(G, V) \leq 14 / 48<1 / 3$. This proves the claim. By Proposition 2.4, $G$ has a regular orbit on $V$, contrary to hypothesis.

Thus $|G: S|=2$. Since every $S$-orbit on $V$ has size at most $p-1$, the preceding paragraph implies that $S$ has a regular orbit on $V$. In particular, $|S| \leq p-1$, and so $p>|S|$. On the other hand, every nonidentity element of $G / \mathrm{Z}(G)$ fixes at most two of the $p+1$ one-dimensional subspaces of $V$. Thus at most 46 such lines are fixed by noncentral elements of $G$. If $|G|=96$, then $p>48$ and so some such line is fixed by no noncentral element of $G$. It follows that $G$ has a regular orbit on $V$, contrary to assumption. Hence $|G|=48$, and so $p>24$. We refine the argument above by observing that the number of lines in $V$ fixed by noncentral elements of $G$ is at most twice the number of prime order subgroups of $G / \mathrm{Z}(G)$. Since $G / \mathrm{Z}(G) \cong \mathrm{S}_{4}$ has 13 prime order subgroups, we see that at most $26<p+1$ lines are fixed by some noncentral element of $G$. As above, this implies that $G$ has a regular orbit on $V$, the final contradiction.

Proposition 3.7. Suppose that $G$ acts imprimitively on $V$. Then $G$ has a regular orbit on $V$.

Proof. We have $V=V_{1} \oplus \ldots \oplus V_{d}$, where $G$ permutes the $V_{i}$ and $\operatorname{dim}\left(V_{i}\right)=1$ for all $i$. Let $C=\mathrm{N}_{G}\left(V_{1}\right) \cap \ldots \cap \mathrm{N}_{G}\left(V_{d}\right)$. Let $V_{i}=\left\langle v_{i}\right\rangle$ for $1 \leq i \leq d$.

Suppose that $d=2$. If every element $x \in G-C$ has order greater than 2 , then $x^{2}$ is a nontrivial element of $C$ for all such $x$. It follows that $v_{1}+v_{2}$ lies in a regular $G$-orbit. We may therefore assume that $G$ contains an involution $t$ that interchanges $V_{1}$ and $V_{2}$. After replacing $v_{1}$ and $v_{2}$ by suitable scalar multiples, we may assume that $t$ interchanges $v_{1}$ and $v_{2}$.

Suppose, for some $\alpha \in \mathrm{GF}(p)^{*}$, that $v_{1}+\alpha v_{2}$ does not lie in a regular $G$ orbit. Then some $y_{\alpha} \in C$ sends $v_{1}+\alpha v_{2}$ to $\alpha v_{1}+v_{2}$, so that $y_{\alpha} t$ fixes $v_{1}+\alpha v_{2}$. Hence $y_{\alpha}$ sends $v_{1}$ to $\alpha v_{1}$ and $v_{2}$ to $\alpha^{-1} v_{2}$. Thus $G$ has a regular orbit on $V$ or $\left\{y_{\alpha}: \alpha \in \operatorname{GF}(p)^{*}\right\}$ is a cyclic subgroup $C_{0}$ of $C$ with $\left|C_{0}\right|=p-1$. Since $v_{1}+v_{2}$ is a regular vector for $C$, our hypotheses imply that $|C| \leq p-1$. Thus $C=C_{0}$. It follows that $v_{1}$ lies in a regular $G$-orbit.

Suppose now that $d=3$. We proceed to estimate the number of pseudoreflections $x$ in each of the 3 or 6 cosets of $C$ in $G$. We note first that $C x$ cannot have order 3 in $G / C$. Indeed the Brauer character $\chi$ of $V$ is induced from a linear character of $\mathrm{N}_{G}\left(V_{1}\right)$. Hence $\chi(x)=0$ and so 1 cannot be a double eigenvalue of $x$ on $V$. Suppose next that $C x$ has order 2 in $G / C$; say $x$ fixes $V_{3}$ and interchanges $V_{1}$ 
and $V_{2}$. Clearly $x$ must centralize $v_{3}$. Suppose that $x$ sends $v_{1}$ to $\beta v_{2}$ and $v_{2}$ to $\gamma v_{1}$ for $\beta, \gamma \in \operatorname{GF}(p)^{*}$. Then $x^{2}$ has $\beta \gamma$ as a double or triple eigenvalue. Since 1 is a double or triple eigenvalue of $x^{2}$, we have $\gamma=\beta^{-1}$ and so $\operatorname{det}(x)=-1$. Now if $y \in C x$ is another pseudoreflection, then $v_{3} y=v_{3}$ and $\operatorname{det}(y)=-1$. Thus $y x^{-1} \in D:=C \cap \mathrm{C}_{G}\left(v_{3}\right) \cap \mathrm{SL}(V)$. We have shown that $C x$ contains at most $|D|$ pseudoreflections. If $g \in G$ does not fix $V_{3}$, then $D \cap D^{g}=1$, and so $|D|^{2}=\left|D D^{g}\right| \leq|C|$. Hence $C x$ contains at most $\sqrt{|C|}$ pseudoreflections. Since $v_{1}+v_{2}+v_{3}$ lies in a regular $C$-orbit, our hypotheses imply that $|C| \leq p-1$ and so $|D| \leq \sqrt{p-1}$.

We claim that $C$ contains either 0 or 3 pseudoreflections. Indeed if $t \in C$ is a pseudoreflection whose matrix with respect to the ordered basis $\left(v_{1}, v_{2}, v_{3}\right)$ is $\operatorname{diag}(\lambda, 1,1)$, then it is easy to see that $\operatorname{diag}(1, \lambda, 1)$ and $\operatorname{diag}(1,1, \lambda)$ are $G$ conjugates of $t$. Hence $\operatorname{diag}(\lambda, \lambda, \lambda) \in \mathrm{Z}(G)$. By Lemma 3.2, however, $|\mathrm{Z}(G)| \leq 2$, forcing $\lambda=-1$. This proves the claim. We note that if $C$ contains 3 pseudoreflections, then they generate a subgroup of order 8 , so that $|C| \geq 8$.

If $|G: C|=3$, then $\operatorname{r}(G, V)=0$ or $\mathrm{r}(G, V)=3 /|G|=1 /|C|$, with the latter possibility occurring only if $|C| \geq 8$. Hence $\mathrm{r}(G, V) \leq 1 / 3$, and so $G$ has a regular orbit on $V$ by Proposition 2.4.

We may now assume that $|G: C|=6$. Let $C<N<G$ with $|G: N|=2$. Then $N$ has a regular orbit on $V$ by the preceding paragraph, and so our hypotheses imply that $|N| \leq p-1$. Thus $|G| \leq 2(p-1)$ and $|C| \leq(p-1) / 3$. Now $G$ contains at most $3 \sqrt{|C|}+3$ pseudoreflections. The number of nonzero vectors in $V$ centralized by some nonidentity element of $G$ is therefore less than

$$
2(p-1)(p-1)+(3 \sqrt{(p-1) / 3}+3)\left(p^{2}-1\right) .
$$

We claim that this quantity is less than $|V|-1$ for $p \geq 11$. That is, we claim that

$$
2(p-1)+(3 \sqrt{(p-1) / 3}+3)(p+1)<p^{2}+p+1
$$

for $p \geq 11$. To verify this inequality, it suffices to show that $3 \sqrt{(p-1) / 3}+3 \leq p-2$ for $p \geq 11$. But the last inequality is equivalent to $3(p-1) \leq(p-5)^{2}$, which indeed holds for $p \geq 11$. Hence $G$ has a regular orbit on $V$ if $p \geq 11$.

Assume then that $p \leq 7$. Then $|G| \leq 2(p-1) \leq 12$. Since $|G: C|=6$, either $|G|=12$ or $|G|=6$. Since the Brauer character $\chi$ of $V$ is an irreducible complex character of $G$, we have $|G|=12$. But then $|C|=2$, so $|G|=12,|\mathrm{Z}(G)| \geq 2$, and some $\chi \in \operatorname{Irr}(G)$ has degree 3. This is impossible. Hence $p \geq 11$ and $G$ has a regular orbit on $V$, as desired.

Proof of Theorem A. By Lemma 2.2, we may assume $P=V$, a $G$-irreducible elementary abelian group of cardinality $p^{d}$. By Corollary 2.5, we may assume that $1<d \leq 3$. Now Theorem A follows from Proposition 3.6 and Proposition 3.7.

\section{REFERENCES}

[1] J. H. Conway, R. T. Curtis, S. P. Norton, R. A. Parker, and R. A. Wilson, Atlas of finite groups, Oxford University Press, Eynsham, 1985. Maximal subgroups and ordinary characters for simple groups; With computational assistance from J. G. Thackray. MR827219 (88g:20025)

[2] J. D. Dixon and A. E. Zalesskii, Finite primitive linear groups of prime degree, J. London Math. Soc. (2) 57 (1998), no. 1, 126-134, DOI 10.1112/S0024610798005778. MR.1624805 (99f:20005)

[3] I. M. Isaacs, Upper bounds for the number of irreducible character degrees of a group, J. Algebra 403 (2014), 201-222, DOI 10.1016/j.jalgebra.2013.12.030. MR3166072 
[4] Olaf Manz and Thomas R. Wolf, Representations of solvable groups, London Mathematical Society Lecture Note Series, vol. 185, Cambridge University Press, Cambridge, 1993. MR,1261638 (95c:20013)

[5] G. A. Miller, H. F. Blichfeldt, and L. E. Dickson, Theory and applications of finite groups, Dover Publications Inc., New York, 1961. MR0123600 (23 \#A925)

Department of Mathematics, Wayne State University, Detroit, Michigan 48202

E-mail address: dgluck@math. wayne.edu 